\title{
A Micro-Phenomenological Lens for Evaluating Narrative Visualization
}

\author{
Stanislaw Nowak* \\ Simon Fraser University \\ Surrey, BC, Canada \\ snowak@sfu.ca
}

\author{
Lyn Bartram ${ }^{\dagger}$ \\ Simon Fraser University \\ Surrey, BC, Canada \\ lyn@sfu.ca
}

\author{
Thecla Schiphorst ${ }^{\ddagger}$ \\ Simon Fraser University \\ Surrey, BC, Canada \\ schiphorst@sfu.ca
}

\begin{abstract}
Narrative visualizations engage audience in data stories, evoking emotions by using narrative patterns, rhetoric, visual design, and content among other strategies. How these elements combine to influence user experiences is complex and difficult to measure using empirical methods. This is partly due to the fact that narrative visualizations influence audiences affectively and implicitly [1][3]. Evaluations of narrative visualizations that aim to better understand these mechanisms should capture this rich complexity by focusing on gathering descriptions of lived experience. Microphenomenology, a rigorous set of methods developed for soliciting descriptions of experiences, has empirically been shown to improve recollection of otherwise implicit aspects of experience [4]. Building on work using micro-phenomenological interviews to evaluate static visualizations [5], we apply these methods to interactive narrative visualizations. We conducted a small study to explore the potential of these methods in this context. Our findings reveal how narrative patterns and designs influence affective states, how they support various forms of exploratory analysis, and how they can facilitate or hinder non-analytical reflection such as the imagining of stories described within visualizations. These types of insights can inform future designs and help researchers understand how techniques employed in narrative visualizations influence users in specific and often implicit ways.
\end{abstract}

Keywords: Information visualization, Visualization design and evaluation methods.

Index Terms: Human-centered computing-VisualizationVisualization application domains-Information visualization; Human-centered computing — Visualization-Visualization design and evaluation methods

\section{INTRODUCTION}

Narrative visualizations aim to engage audiences, persuade, and evoke affective responses [3], [6], [7]. Critical to these types of visualizations is the evoking of an experiential response that is beyond simply comprehending the data values. Narrative visualization designers use elements like narrative patterns and structures [2], [6], rhetorical devices [1], and other design features like color and animation [3] to achieve their communicative intent.

\footnotetext{
*e-mail: snowak@sfu.ca

†e-mail: Iyn@sfu.ca

te-mail: schiphorst@sfu.ca
}

These elements combine in ways that complicate the evaluation of these experiential scenarios [8]. Traditional empirical evaluation methods and metrics are insufficient for capturing this richness; visualization researchers are turning to qualitative methods that are well-suited to capture the complex and holistic nature of experiences [8] [9].

We present micro-phenomenological methods (henceforth $M P M)$ for interview and data analysis as a way to evaluate narrative visualizations [10]. The MPM elicitation interview $(E I)$ is a rigorous interview technique that is designed to guide interviewees to re-enact an experience mentally, become aware of specific aspects of their subjective experiences, and describe them with precise detail [11]. MPM can help users to become aware of implicit decision-making processes and improve the accuracy with which decision-making processes are recollected [4]. Narrative visualizations use techniques that may influence implicit aspects of user's experiences. Researchers have described how rhetorical techniques in visualizations may connote various meanings to audiences and thus bias, or frame, their interpretations in implicit ways [1]. Using MPM in this context may offer insights into how narrative visualizations influence experiences at an implicit level.

EI was specifically designed to minimize biases, like postrationalization, that are often involved in introspective interviews [12]. This sets EI apart as a method that has the potential to solicit richer and more accurate descriptions of experience.

EI is able to help interviewees gain awareness of implicit aspects of experience by focusing on the physical or mental procedural actions [11]. Rather than focusing on the "what" or "why" questions, EI emphasizes the "how" [11]. Often, when engaged in a task such as viewing a data visualization, one is attending to the content of what they are viewing and not how they are making sense of the information presented. This state of full devotion to a task characterized by a lack of meta-cognitions has famously been described as "flow" by Csikszentmihalyi [13]. It is a state that is closely tied to the experience of data stories [2]. By focusing attention on these procedural "how" aspects of experience, EI has the capacity to reveal otherwise unavailable information about complex flow-types of experiences. These implicit aspects of experience, which can be accessed retroactively, are described to be a part of pre-reflective consciousness [11]. By using MPM, researchers and designers can gain insight into how users experience and interpret narrative visualizations instead of relying on assumptions.

We conducted a small study exploring MPM as an evaluation technique for narrative visualizations. Our research builds on the work of Hogan et al. who introduced EI as an evaluation tool to visualization by way of a study evaluating static visualizations [5]. We expand this research by applying this methodology to interactive narrative visualization. We posit that narrative visualizations, by employing strategies such as animation and interactive exploration, create a sense of flow. The capability of 
MPM to reveal implicit aspects experiences make it an ideal tool for studying techniques used in narrative visualizations such as those intended to create a sense of flow. Our findings show how MPM can be used as an experiential narratological tool revealing how narrative patterns are experienced in the context of their unfolding, how affective, somatic, and cognitive processes intertwine and guide the exploratory process, and how participants are provoked to reflect in diverse ways by various design facets of narrative visualizations.

\section{BACKGROUND}

\subsection{Affect and Experience in Visualization}

Narrative visualizations aim to increase user engagement and evoke affective responses [3], [6]. Affect matters for communicative intent and engagement [3], [6] and plays important roles in visualization [14]-[17]. Eliciting affective states enhances problem solving and reduces boredom and frustration [14], [18]. Researchers have shown how visualizations can prime affective states and how design features such as color and motion can possess rich affective expressiveness [14]-[16].

Affect and cognition are the two interdependent information processing systems used by humans [19][20]. The interplay of these systems is a critical part of processes in complex tasks involving intuition [21]. Individuals conducting tasks that involve intuition often report being guided by physical (somatic) sensations and general feelings (affective)[21]. Interactive visual exploratory analysis is one such task involving intuition [22] and is often present in narrative visualizations [6]. These two systems are also present in user engagement which has been defined as the "emotional, cognitive and behavioral connection that exists, at any point in time and possibly over time, between a user and a resource" [23]. Understanding how a resource, such as a narrative visualization, influences a user's engagement and exploratory experience requires methods of study that capture the richness and detail of these relationships.

\subsection{Comparison to other methods}

MPM and EI complement and extend other traditional qualitative methods used in Human-Computer Interaction (HCI) and visualization research for studying subjective experiences [5]. When compared to MPM, however, observation, diaries, interviews, and questionnaires either do not capture rich enough data about personal experiences or suffer from introducing potential bias from post-rationalization [5].

Situated recall methods, such as Cued-recall Debrief (CRD), have been used to accurately report when affective changes occur and describe some of associated details of an experience in the context of an HCI system [24]. CRD and other situated recall methods may be useful in identifying when affective changes occur but may not be as capable of providing as rich and reliable data as MPM. As suggested by Hogan et al., CRD has the potential for introducing post-rationalization and confabulations as it does not follow the same rigorous process as MPM [5]. The emphasis on procedural aspects of experience make MPM a more suitable method for soliciting descriptions about experiences dealing with tasks such as viewing a data visualization.

\subsection{Micro-Phenomenology}

MPM is a set of interview and analysis methods that focus on gathering descriptions of lived experiences with a high degree of accuracy and reliability [10]. The methodology consists of EI and a method for analysis and validation of the data [10]. Originally developed by Vermersch for understanding cognitive processes in learning [12], [25], these methods have since been applied to a variety of contexts including HCI [26], the study of intuitive experiences [27], subjective experiences in anticipation of epileptic seizures [28], and more recently static visualizations [5].

The MPM approach finds its strength in focusing on procedural aspects of experience. Accessing procedural memories is difficult, despite an intuitive and naïve belief that awareness of our own experiences is straightforward [28]. This is perhaps why individuals are prone to post-rationalize their experiences. An empirical study introducing EI to a classic experiment of "choice blindness", which has traditionally been used to discredit introspective methods, demonstrates the strengths of MPM [4]. In the study, participants were asked to choose one out of two pictures of women who they found to be more attractive. After several trials, participants were presented with the photo they chose and asked to explain their decision-making process. In some of the trials, participants were tasked with explaining a choice they had not made. In the original design of the study, participants detected the manipulation in $33 \%$ of the trials. In another version, where EI techniques were introduced, detection of the manipulation increased to $80 \%$. This study demonstrates how our awareness of experiences tends to be poor, but can be helped with proper guidance [4] and highlights how MPM can improve the accuracy of qualitative interview studies while revealing details of which one would normally be unaware. In the context of narrative visualization, where designs may influence user experiences and bias interpretation in various ways, insights about procedural and implicit aspects of experience may reveal valuable information that could be used to inform the design of future visualizations.

\subsection{MPM in visualization}

Hogan et al. introduced EI and MPM to visualization in a study that focused on static visualizations to explore the potential of EI as an evaluation tool. [5]. They demonstrated how this technique can be used to gather accounts of lived experiences that capture hedonistic, emotional, and sensory content, and reveal sensemaking processes, personal reflections, and meaning-making processes [5]. The authors suggested future research should focus on exploring EI in interactive visualization contexts [5], as . interaction introduces entirely new dimensions to experiences of visualization, particularly in the context of authored narrative visualizations. Interactions give users a sense of agency, yet can be constrained in a way that steers them to interact with predetermined subsets of data that may influence their interpretations [2].

\section{Method}

Our approach is inspired by Hogan et al. for application in narrative data visualizations [5]. Differences in procedure from those suggested by Hogan et al. and their rationale are described in section 4.3. The following section provides a general description of the structure of EI.

The interview procedure follows a series of iterative steps: (1) selecting a particular experience, (2) evoking the experience, (3) inquiry into the diachronic (temporal) dimension of experience, and (4) inquiry into the synchronic (deepening of awareness and description) dimension [11].

The first step, (1) selecting a particular experience, involves identifying a single lived experience rather than a general experience. For example, MPM would not be applied to the study of one's experiences of data visualizations throughout their lifetime, but rather a particular instance of viewing a data visualization [11].

The next step, (2) evoking the experience, begins the interview process. At the start of and throughout the interview, participants should be re-informed about the nature of the interview and asked for permission to inquire about a given aspect of experience. The 

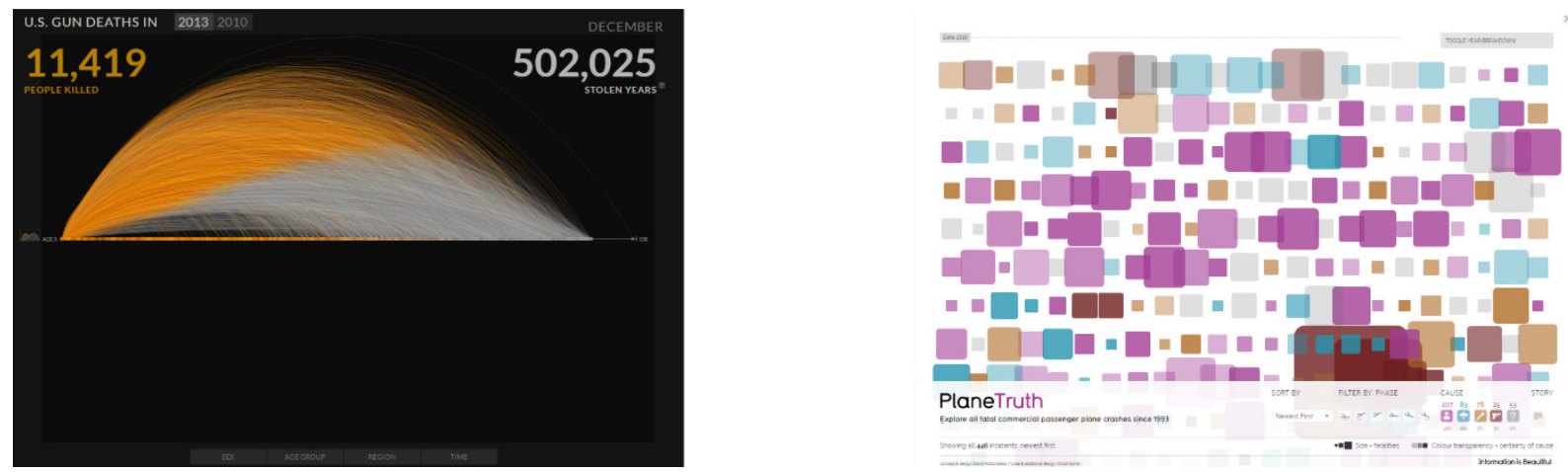

Figure 1: Read from left to right. (A) "U.S. Gun Deaths" by Periscopic [29], (B) "Plane Truth" from Information is Beautiful [30]

participant is to be made aware about the iterative and in-depth nature of the interview and their right to withdraw at any point: "If you agree, I would like to go back to your experience of X. How did you feel at this time?"

To induce a state of evocation, a state where the participant reenacts the experience in question whilst in a state of deep introspection, the interviewer asks questions relating to sensory and affective aspects of the experience: "Could you go back to the moment when you sat down to view the data visualization? What do you see? What do you smell? What is around you? How do you feel? " It is critical to ask questions in the present tense as it aids interviewees to place themselves in the re-enactment of the experience. The state of evocation is recognizable by certain cues such as the glazing over of eyes, gazing into space, taking long pauses in speech, slurring speech, and speaking in the present tense [11].

Step (3) inquiry into the diachronic dimension focuses on guiding the interviewee to reveal the temporal unfolding of the experience at hand. This is facilitated by asking open-ended "empty-content" questions about the experience: "What happens next?" [29].

After the diachronic dimension has been described, the interviewer focuses on deepening the description of experiential details that are interesting and relevant to the research at hand. This leads to step (4), inquiry into the synchronic dimensions. This involves iterative questioning where participants are repeatedly asked to point their attention to a specific aspect of experience, evoke it, and deepen their awareness of its procedural components [11]. Often vague words and para-verbal cues will be asked about for clarification. Interviewers will ask questions like: "You mentioned you did X. You said you feel sad. How do you know you feel sad?".

\subsection{Data Analysis}

Once interviews are completed, transcripts of the interviews are analyzed using a variety of potential qualitative analysis methods [5]. Using video, audio, and text, transcripts are searched to identify instances where participants were or were not in a state of evocation. Indicators exist in the form of verbal, gestural, and paraverbal cues. Often, this is visible in the content of their speech. One example is the casting of judgement about the experience. The following quote demonstrates such a case. It is marked in bold and underlined: "I couldn't really figure it out for like... a minute until I saw the question mark box by the stolen years figure... and I guess I was kind of satisfied by that explanation. Although, I think it's a bit weird... and a bit extrapolaty... still not entirely sure what they did there." [P1 Fig. 1.A.]. Upon identification of states where participants are not in a state of evocation, these records are removed to reduce the transcript to only contain records where participants are describing their experiences as they happened [5]. These records are then analyzed using a qualitative analysis method such as thematic analysis [5].

\section{The STUDY}

The goal of our study was to understand how MPM might apply to the evaluation of interactive narrative visualizations. As part of our study we sought to answer how MPM could serve as an effective evaluation technique for narrative visualizations and what unique insights about techniques employed in narrative visualizations it could reveal.

We chose two contrasting interactive narrative visualizations for our study. The first is the "U.S. Gun Deaths" visualization by Periscopic (Fig. 1.A.), which depicts gun-related deaths in the United States in the years 2010 and 2013 [30]. The visualization has been praised for being cinematic and emotionally evocative [3]. The visualization features a dramatic introductory animation sequence. This opens to an interface allowing users to freely explore filters across demographic dimensions and view tooltips, which provide details about causes of death and expected lifespans.

The second visualization is "Plane Truth" from informationisbeautiful.com (Fig. 1.B.) [31]. This visualization depicts data about the plane crashes. This visualization is less authored and more exploratory. It did not use, from our perspective, as many discernable design decisions intended to steer a narrative. Additionally, we felt that the design of the visualization was lighthearted and less emotionally evocative. We chose these two contrasting visualizations to capture experiences of techniques utilized in both more author-driven and more user-driven approaches in narrative visualization [6].

\subsection{Participants}

Six participants ranging between 20 and 30 years of age took part in the study. Five were male and one was female. The participants had varying degrees of data literacy and came from a variety of professional backgrounds including painting, therapeutic counselling, interaction design, software engineering, neuroscience, and biotechnology. Participants were preferentially selected if they had some form of introspective practice such as meditation or cognitive behavioral therapy to facilitate the interview process and introspective exercise.

\subsection{Procedure}

3 participants viewed each of the selected visualizations. Participants viewed the visualization for up to 20 minutes or as long as they took to feel satisfied in their exploration. Shortly following, 

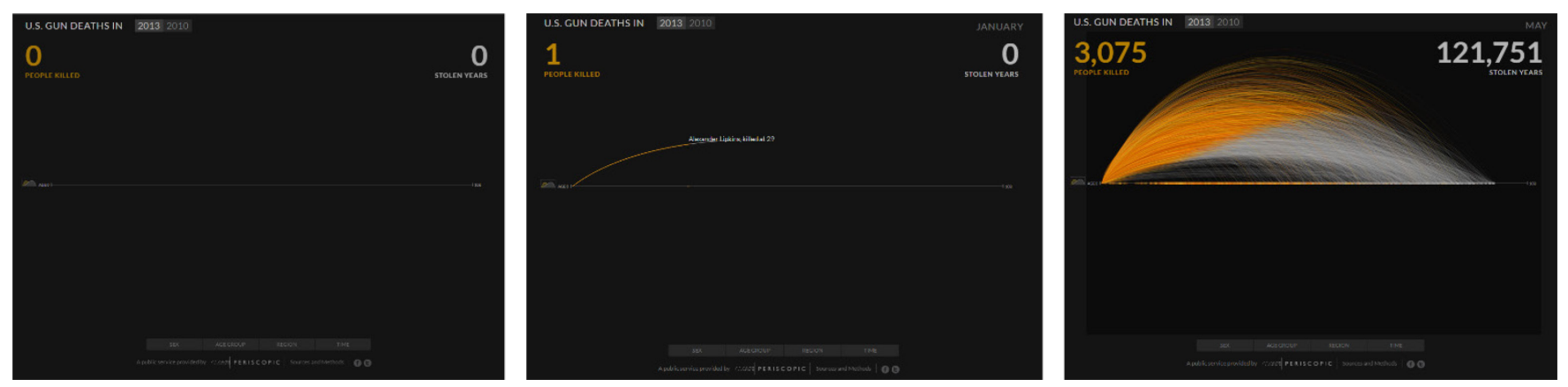

Figure 2: Read from left to right. (A) fade in of axis, (B) first arc is drawn, (C) animation accelerates as multiple arcs are drawn [29]

they conducted a short breathing exercise to facilitate an introspective state of mind. Thereafter, the interview began. All interviews were conducted by the same researcher. 4 interviews were conducted in person, while 2 were conducted using remote video conferencing software. Locations for interviews were selected based on appropriateness of atmosphere for the interview and convenience.

Prior to the session start, participants were briefed on the nature, purpose, and methods of the interview. Participants were asked consent to proceed with the study. Sessions were video and audio recorded. The recordings were transcribed by the same researcher who conducted the interviews. Each interview lasted between 30 and 50 minutes.

Interviews followed the format described in section 2.3.1. Interviews were transcribed from video and reduced to only include records of evocation as described in section 2.3.2. This initial transcription also allowed for deep immersion into the data. Once the videos had been transcribed and reduced to records in which participants were in a state of evocation, they were analyzed using thematic analysis [32] as suggested by Hogan et al. [5]. Videos were transcribed and analyzed using NVIVO software [33]. Thematic analysis generally follows several phases. The approach we took for our analysis is described below.

\subsubsection{Familiarization}

As an initial phase, transcripts were read for familiarization of the data. This approach allows researchers gain an overview of the data in order to further aid the coding process. At this stage, a start list of potential codes that may be evident within the data was identified.

\subsubsection{Thematic Coding}

As a first pass, the data was coded according to an open coding paradigm. Line by line, all the data was coded according to identified topics. The codes were iteratively refined to arrive at an exhaustive list of thematic codes. This stage involved roughly 6 coding passes.

\subsubsection{Searching for, reviewing, and defining themes}

The last stages of the analysis include searching for common summative themes within and between participants across codes. Themes are broader than codes and encompass information that is more detailed and closer to addressing research questions and purposes. These themes were reviewed and refined until a final set emerged. These themes were then defined and named. This final stage took close to 4 iterations.

\subsection{Unique adaptations of procedure}

As part of our approach, we modified some of the procedure suggested by Hogan et al. The primary difference in our approach was that we had participants view visualizations in the same session as the interview. This was partially due to scheduling convenience, but primarily it was to ensure an environment with limited distractions to maximize potential engagement with the visualizations. A limitation of this approach is that it may bias the experience. Indeed, one participant expressed that they were sometimes guided by a sense of what was expected of them instead of what their natural inclinations were. This bias may also, however, be present in studies that allow participants to explore visualizations on their own time. We felt that this bias could be effectively mitigated by communicating the nature of the study to participants and thought it more important to ensure a distractionfree environment.

An additional adaptation was the use of a breathing exercise. The choice to include a breathing exercise was made to bridge the viewing and interview stages of the sessions. The breathing exercise encourages a change in mental state to help participants become more mindful and re-direct attention towards internal aspects of their experience. Past research suggests that mindfulness training techniques, such as breathing exercises, improve abilities of endogenously orienting attention [34]. MPM depends on the interviewee's ability to redirect their attention to aspects of their experience that would otherwise go unnoticed. We felt this was especially important when transitioning from the task-oriented activity of exploring a visualization to the introspective activity of an interview.

Many breathing exercise techniques have been established. In mindfulness training the objective is to point attention to one's own breathing. Our "square" breathing exercise consisted of 4-second intervals for inhalation, exhalation, and holding one's breath between inhales and exhales.

We found maintaining an appropriate mindset for EI was critical in soliciting rich and insightful data. The adaptations we chose to make were primarily intended to facilitate these types of mental states.

\section{Results}

Our results build on the findings of Hogan et al.[5]. Many of the themes found in a general application of MPM to static visualizations were also found in our study. Our study reveals further types of insights that could be gleaned from using MPM in narrative visualizations. In particular, we found MPM effective at capturing aspects of "flow", which are critical to data stories [2], in ways that other methods 
Table 1: List of themes extracted from analysis. Numbers in parenthesis signify how many participants the code was applied to.

\begin{tabular}{|c|c|c|}
\hline $\begin{array}{l}\text { Experiential Narratology } \\
\text { Anticipation (3), Initial Understanding (2), Full } \\
\text { Understanding (2), Boredom (2) }\end{array}$ & $\begin{array}{l}\text { Experiences that accompany the unfolding of narrative } \\
\text { structure such as an opening animation sequence. }\end{array}$ & $\begin{array}{l}\text { "Then the trajectory dropped... the individual was } \\
\text { killed, and the indicator plummeted } \ldots \text { and I understood } \\
\text { the nature of the visualization" }[\mathbf{P 1} \text { Fig. } 2 . \mathrm{A}]\end{array}$ \\
\hline $\begin{array}{l}\text { Exploratory Processes } \\
\text { Urges (2), Somatic (4), Personalizing Data (3), Previous } \\
\text { Knowledge (4), Affective (6) }\end{array}$ & $\begin{array}{l}\text { Thoughts, affective states, and physical sensations that } \\
\text { arise during exploration. }\end{array}$ & $\begin{array}{l}\text { "I wanted to keep reading them (stories in tooltip), but } \\
\text { then I thought I should try to figure out the other part of } \\
\text { the chart" [P5 Fig. 1.B] }\end{array}$ \\
\hline $\begin{array}{l}\text { Experiencing Hypothesis Testing } \\
\text { Confirming Expectations (2), surprise (3), relief (1), } \\
\text { contradicting expectations (1) }\end{array}$ & Experiences of expectations being or not being met. & $\begin{array}{l}\text { "I guess in my head that the majority of people dying } \\
\text { from guns was going to be in the 0-18 (age) range... but } \\
\text { when Istarted with the highest adult age... It was higher } \\
\text { than I thought it would be" [P3 Fig. 1.A] }\end{array}$ \\
\hline $\begin{array}{l}\text { Trust or Belief } \\
\text { Acceptance (1), belief (2), intellectual superiority (1), } \\
\text { skepticism (4) }\end{array}$ & $\begin{array}{l}\text { Sensations relating to trust and belief regarding authors, } \\
\text { data, or arguments. }\end{array}$ & $\begin{array}{l}\text { "When I saw there wasn't a y-axis I felt a little worried } \\
\text { that I was being misled or massaged towards a certain } \\
\text { conclusion" [P1 Fig. 2.A] }\end{array}$ \\
\hline $\begin{array}{l}\text { Engagement } \\
\text { Interest (3), disinterest (4), boredom (2) }\end{array}$ & $\begin{array}{l}\text { Experiences during viewing of visualization that } \\
\text { signaled engagement and interest in the visualization. }\end{array}$ & $\begin{array}{l}\text { "I actually feel more engaged because like my whole, } \\
\text { body is interacting in order to find out that solution..." } \\
\text { [P4 Fig. 1.B] }\end{array}$ \\
\hline $\begin{array}{l}\text { Sense-making processes } \\
\text { Comparison (3), Relating Data (3) }\end{array}$ & $\begin{array}{l}\text { Processes in which information from visualization is } \\
\text { mentally contextualized to make meaning. }\end{array}$ & $\begin{array}{l}\text { "What else is } 15000 \text { years? Can I split that quantity up } \\
\text { into something? What do I know that's like } 15000 ? \\
\text { Dinosaurs popped into my head for a second..." [P2 } \\
\text { Fig. } 2 . \mathrm{C}]\end{array}$ \\
\hline $\begin{array}{l}\text { Reflection processes } \\
\text { Conjecture theorizing (3), Current Events and Previous } \\
\text { Knowledge (4), Personal Experiences (2), Imagination } \\
\text { and Mental Visualization (4) }\end{array}$ & $\begin{array}{l}\text { Reflecting on the data through personal memories, } \\
\text { knowledge about current events, internal imagination of } \\
\text { stories told by data, or conclusions, questions, or } \\
\text { conjectures reached from viewing the visualization. }\end{array}$ & $\begin{array}{l}\text { "I was picturing the plane flying... and the cabin... and } \\
\text { the two captains passed out, but the plane just flying." } \\
\text { [P5 Fig. 1.B] }\end{array}$ \\
\hline $\begin{array}{l}\text { Experiences of Aesthetics and Design } \\
\text { Embodiment (2), Hedonics (4), Perceived Agency (2) }\end{array}$ & $\begin{array}{l}\text { Perceptions of aesthetics and design of visualization that } \\
\text { include hedonics, the embodiment of felt affect in the } \\
\text { visual design, and perceived agency of the visualization. }\end{array}$ & $\begin{array}{l}\text { "The leading edge of the line... it like drops to the axis } \\
\text { and that was a sad feeling. Kind of like a visceral } \\
\text { animation for the actual feeling of the person dying". } \\
\text { [P2 Fig 2.B] }\end{array}$ \\
\hline $\begin{array}{l}\text { Experiencing the data } \\
\text { Dissatisfaction (1), Affective Dissonance (3), disturbed } \\
\text { (1) }\end{array}$ & Experiences of data when reflecting on it. & $\begin{array}{l}\text { "The one that was funny to me was like 'endocrine } \\
\text { disease'. I was like that's the most impersonal thing to } \\
\text { relate for me... I wanted something more personal in } \\
\text { those boxes." [ } \mathbf{P 2} \text { Fig } 1 . \mathrm{A}]\end{array}$ \\
\hline
\end{tabular}

might not have been able to. We found that EI can capture rich emotional and sensory experiences and reveal sense-making processes, personal reflections, and meaning-making processes that occur when viewing narrative visualizations. Table 1 lists the various themes we identified in our analysis. These exemplify the types of findings that MPM may reveal in evaluating narrative visualizations.

\subsection{Experiential Narratology}

Participants described the experiential unfolding of the opening animation sequence of the "U.S. Gun Deaths" visualization. This animation capitalizes on two narrative patterns identified by

researchers [2]. The first is a gradual reveal where the animation progressively reveals more information to decode the visualization, understand the nature and argument of the visualization, and set the pace and rhythm of the narrative flow [2]. This is coupled with a speeding up of the animation which creates a sense of urgency and dramatic tension [2]. The following descriptions illustrate the experiential stages that accompany the unfolding of this animation.

At the outset of the animation sequence, when axes slowly fade in against a dark background, participants felt a sense of anticipation as to what was going to happen next in the visualization (Fig. 2.A). This is illustrated by the following quote: "It was kind of game-like. Kind of fun... I knew it was about gun deaths. I was waiting for the message to sink in." [P1 Fig. 2.A]

At the next stage of the animation an arc flies across the window terminating at a circle mark indicating the lifespan of an individual
(Fig. 2.B). A name is presented with the age at which they were killed in a gun-related incident. Participants felt an initial sense of understanding of the data: "Then the trajectory dropped... the individual was killed, and the indicator plummeted... and I understood the nature of the visualization" [P1 Fig. 2.B]. Participants felt that the animation embodied an affective expression. "The leading edge of the line... it like drops to the axis and that was a sad feeling. Kind of like a visceral animation for the actual feeling of the person dying". [P2 Fig. 2.B] reflecting earlier evidence that motion influences affective impressions [16]

More arcs are drawn at an accelerating rate to reveal the aggregated data of gun-related deaths and a derived metric, "stolen years", using life expectancy data to estimate the number years that individuals could have lived had they not been killed in a gunrelated incident (Fig. 2.C): "Then multiple trajectories started coming out and accelerated like crazy until the whole graph started turning orange with those lines that then plummeted and started turning grey. When that happened that was like a wow moment... I felt super shocked... it was simultaneously shocking to see that information presented visually. " [P1 Fig. 2.C]

Once the animation sequence had finished, participants reported attempts to understand how the "stolen years" metric was derived and ways of making the large number more meaningful to themselves. This sense-making process is illustrated by the following quote: "What else is 150000 years? Can I split that quantity up into something? What do I know that's like 150000? Dinosaurs popped into my head for a second." [P2 Fig. 2.C.]. 
These findings highlight how MPM can provide highly detailed descriptions of experiential states accompanying the unfolding of a narrative. The descriptions can reveal how the animation sequence, the pace of information delivery, and design choices such as motion can accompany, embody, and evoke affective states as well as create a narrative flow. Additionally, internal sense-making processes help illustrate how an audience may make meaning of out of the information presented. Insights into these mental operations can inform future designs that aim to evoke a certain type of experience or facilitate meaning-making.

\subsection{Exploratory Processes}

Another narrative pattern which aims to increase engagement is exploration [2]. Users may feel a sense of engagement through being in control of the story's content and unfolding [2]. If the possibilities of exploration are left too open, users may become disengaged [6]. As a result, understanding how to constrain exploration is critical for maintaining engagement [2]. Capturing user experiences of exploration may reveal ways in which interactive mechanisms and curiosity combine to engage or disengage users. Both the "Plane Truth" and "U.S. Gun Deaths" visualizations employed exploration in a way that engaged participants.

Often, guiding participant's exploration was interest in personally relevant data: "I want to know (about) where I am from... and I clicked on the Northwest and I thought people must be more happy..." [P3 Fig. 1.A]. Others were curious about previous knowledge they held: "I saw two sort of dark red rectangles, and then when I saw that I thought 'Oh yeah, probably that has something to do with plane crashes'. I thought it was 9/11" [P6 Fig. 1.B]. Understanding the interests driving exploration can inform future designs by making designers aware of the various questions that could be asked and how to best cater to those as analytical tasks.

In certain instances, participants felt urges guiding their exploration. One participant was captivated by the aesthetic design of the square marks in the "Plane Truth" visualization. They felt that the design was inviting them to interact: "Something within me wanted to know what would happen." [P4 Fig. 1.B]. Another participant perceived the responsiveness of the square marks as the marks wanting him to click on them "I toggle the mouse over the squares. They got bigger or brighter... like they wanted me to click on it" [P5 Fig. 1.B]. This illustrates how aesthetics could be used to steer participants to certain types of interaction and thus author the exploratory process.

During interviews, participants became aware of somatic feedback. One participant viewing the "Plane Truth" visualization described becoming aware of their own engagement with the visualization by taking note of how their body was leaning forward close to the screen. They were trying to discern slight differences in color transparency used to encode the certainty associated with reports of causes of plane crashes: "I actually feel more engaged because... my whole body is interacting in order to find out that solution" [P4 Fig. 1.B]. While in this case the participant felt a sense of engagement, this description highlights a potential usability issue. Another participant tied the importance of a surprising insight to a physical sensation: "It's like some kind of heat wave... goes through your body or head... It's kind of a mindblowing feeling." [P2 Fig. 1.A]. A participant in a different instance became aware of their frustration when they felt they were being misled: "There is this annoying tension between my stomach and heart area." [P3 Fig. 1.B]. This description also illustrates instances where trust and belief may be affected by strategies employed in narrative visualizations.

Participants often felt their affective state change as the exploratory analysis progressed. One participant felt a sense of pride or satisfaction at having correctly had their expectations met: "I started thinking about how that kind of makes sense based on my experiences and what I know about the area... there was a twinge of pride or some kind of satisfaction or pleasure..." [P1 Fig. 1.B].

Our findings reveal various affective, somatic, and cognitive experiences involved in exploring visualizations and how they may be supported or influenced by various strategies. Our data illustrate how aesthetics and responsive design, as seen in the "Plane Truth" visualization, can support engagement. Additionally, our findings reflect how personally relevant data, personal interests, and ego play a role in exploratory processes.

\subsection{Reflection Processes}

Exploratory experiences were often non-analytical. Instead, participants engaged in various forms of reflection. This included reflections about personal experiences: "The landing, kind of like an impact. That's unsettling obviously. Something that resonated with that experience from a week ago was when I was looking at planes that were in flight, in landing, or in taxi" [P6 Fig. 1.B]. It also included reflections about current events and previous knowledge: "I am relating to news stories to similar things happening. I was relating it to gun deaths in Chicago... so just kind of relating it to people who I had seen interviewed or read about in stories" [P2 Fig. 1.A].

Participants mentally visualized the stories that were presented to them. This mental imagining was prompted by short descriptions present in tooltips within both visualizations: "I was picturing the plane flying... and the cabin... and the two captains passed out, but the plane just flying." [P5 Fig. 1.B] A participant viewing the "U.S. Gun Deaths" visualization lamented that they did not feel the sense of relief that they desired from the description: "I didn't feel like I got the relief that I wanted from the second bubble", [P2 Fig. 1.A]. They felt that content in another tooltip was more relatable and easier to imagine "It felt less like a statistic and more like a setting you could place" [P2 Fig. 1.A]. These descriptions reflect a narrative pattern called humans-behind-the-dots which aims to build empathy in users through individual concrete examples of the main story [2]. These findings reflect how the type information presented can facilitate or hinder empathy and imagination in users.

Narrative visualizations facilitate non-analytical reflections about personal lives, previous knowledge, and the stories contained within the data. Our findings demonstrate how data contained within elements such as tooltips can facilitate, or hinder one's imagining of stories.

\section{Discussion}

We found that descriptions of the "U.S Gun Deaths" visualization more frequently described experiences related to what could be considered intentionally authored narrative techniques. With some important exceptions, a substantial portion of our data relating to the "Plane Truth" visualization focused on decoding the visualization and usability issues. This may reflect the differences in the levels of authoring in each visualization. It could potentially be due to the limited time allotted for participants to explore visualizations, but it also serves to highlight MPM's strengths and weaknesses in evaluating narrative visualizations. MPM is useful for understanding how narrative visualizations accomplish their communicative intent but may be too laborious and time-intensive for evaluating visualizations as they are being developed.

Our study demonstrates the types of insights that can be gleaned from using MPM for evaluating narrative visualizations. These methods can provide rich descriptions of experiences, which may have been occluded had an alternative method been implemented. This serves to reveal how various data storytelling techniques such 
as narrative patterns or rhetoric support the communicative intent of a narrative visualization. The method is strong in preserving realism. Using MPM on its own or in combination with other methods can shed light on how techniques used in visualizations achieve their communicative intent and thus inform future designs.

While MPM has been shown to improve the reliability of introspective inquiry, the validity of results in individual studies is difficult to establish [35]. Researchers have suggested methods to address this issue [36].

We have found that MPM methods are difficult to execute as they rely in large part on the willingness of participants to share deep aspects of their experiences. Repeatedly iterating over and delving deep into experiences can leave participants feeling uncomfortable. To facilitate a fruitful interview, we found that creating a relaxed and comfortable atmosphere while building trust and a sense of security with the participant were essential. We found that providing a convenient meeting time and space ensured that participants were fully present and engaged in the experiment. On several occasions sessions were postponed due to participant's work or life-related stresses, which may have served to distract or hinder a useful interview session. Additionally, controlling the viewing and interview environment and conducting the mindfulness-training inspired breathing exercises further aided participants in reaching a demeanor that was amicable to evocation.

We found that while single 30-60 minute sessions, as demonstrated in the work in Hogan et al., were sufficient to gain rich descriptions of the experience, conducting longer and repeated interviews as in other micro-phenomenological studies [27] may provide deeper and more exhaustive insights.

We suggest that future studies utilizing MPM may want to separate the interview into two stages as suggested by the designers of the method [11]. The two stages being the establishing of the temporal structure of the experience and the deepening of the description for the experience. Separating the interview into two sessions would allow interviewers to review the transcript and identify points of interest to follow-up on in the second interview. The single session structure that our research followed required the interviewer to identify interesting aspects of the experience to follow-up on in real-time. This is relatively difficult and may result in an interview that may not be as thorough and exhaustive. An additional benefit of this structure is that participants are less likely to become fatigued from the long and intensive interview sessions.

\subsection{Limitations}

Our research has only focused on one case-study of using MPM in a narrative visualization context. Our reflections on MPM as a tool used for narrative visualizations are limited to the perspective of this single case-study. Additionally, coding and analysis of transcript was limited to one researcher. Nevertheless, we believe that the findings from this study demonstrate the affordances of MPM in evaluating narrative visualizations and can be extended to other domains in visualization which have similar aims.

\section{Conclusion}

We have demonstrated how MPM could be used as a tool for evaluating narrative visualizations. Our findings show that it may be used for revealing experiences of narrative patterns, how visual rhetoric and design choices can embody and accompany affective experiences, how the somatic, affective, and cognitive systems interact throughout the exploration of a narrative visualization, and how individuals reflect on and make sense of data. Our study touched on questions primarily involved in the user experience evaluations scenario, but also demonstrated how it may be used in the communication through visualization and visual data analysis and reasoning scenarios [9]. Our contributions in this paper are the introduction of MPM for evaluation narrative visualizations and a demonstration of and findings from a study using MPM for evaluating narrative visualization.

Future research may focus on further adapting microphenomenological methods to visualization and developing ways of validating findings. One possible avenue for validation would be triangulation using other sources of data.

\section{ACKNOWLEDGEMENTS}

This work was supported by the National Sciences and Engineering Research Council of Canada.

\section{REFERENCES}

[1] J. Hullman and N. Diakopoulos, "Visualization Rhetoric: Framing Effects in Narrative Visualization," IEEE Trans. Vis. Comput. Graph., vol. 17, no. 12, pp. 2231-2240, Dec. 2011.

[2] N. H. Riche, Ed., Data-driven storytelling. Boca Raton, Florida: Taylor \& Francis/CRC Press, 2018.

[3] A. Cairo, "U.S. Gun Deaths and the Callenge of Uncertainty," Peachpit, 2013.

[4] C. Petitmengin, A. Remillieux, B. Cahour, and S. CarterThomas, "A gap in Nisbett and Wilson's findings? A firstperson access to our cognitive processes," Conscious. Cogn., vol. 22, no. 2, pp. 654-669, 2013.

[5] T. Hogan, U. Hinrichs, and E. Hornecker, "The Elicitation Interview Technique: Capturing People's Experiences of Data Representations," IEEE Trans. Vis. Comput. Graph., vol. 22, no. 12, pp. 2579-2593, Dec. 2016.

[6] E. Segel and J. Heer, "Narrative Visualization: Telling Stories with Data," IEEE Trans. Vis. Comput. Graph., vol. 16, no. 6, pp. 1139-1148, Nov. 2010.

[7] R. Kosara and J. Mackinlay, "Storytelling: The Next Step for Visualization," Computer, vol. 46, no. 5, pp. 44-50, May 2013.

[8] S. Carpendale, "Evaluating Information Visualizations," in Information Visualization, vol. 4950, A. Kerren, J. T. Stasko, J.-D. Fekete, and C. North, Eds. Berlin, Heidelberg: Springer Berlin Heidelberg, 2008, pp. 19-45.

[9] H. Lam, E. Bertini, P. Isenberg, C. Plaisant, and S. Carpendale, "Empirical Studies in Information Visualization: Seven Scenarios," IEEE Trans. Vis. Comput. Graph., vol. 18, no. 9, pp. 1520-1536, Sep. 2012.

[10] "micro-phenomenology | Home," micro-phenomenology. [Online]. Available: https://www.microphenomenology.com/home. [Accessed: 10-Feb-2018].

[11] C. Petitmengin, "Describing one's subjective experience in the second person: An interview method for the science of consciousness," Phenomenol. Cogn. Sci., vol. 5, no. 3-4, pp. 229-269, Dec. 2006.

[12] P. Vermersch, L'entretien D'explicitation. ESF, 1994.

[13] M. Csikszentmihalyi, "Flow: The Psychology of Optimal Experience," 1990.

[14] L. Harrison, "The role of emotion in visualization," Ph.D., The University of North Carolina at Charlotte, United States -- North Carolina, 2013.

[15] L. Bartram, A. Patra, and M. Stone, "Affective Color in Visualization," in Proceedings of the 2017 CHI Conference on Human Factors in Computing Systems, New York, NY, USA, 2017, pp. 1364-1374. 
[16] M. Lockyer and L. Bartram, "Affective motion textures," Comput. Graph., vol. 36, no. 6, pp. 776-790, Oct. 2012.

[17] C. Feng, L. Bartram, and B. E. Riecke, "Evaluating Affective Features of 3D Motionscapes," in Proceedings of the ACM Symposium on Applied Perception, New York, NY, USA, 2014, pp. 23-30.

[18] R. W. Picard, Affective computing. Cambridge, Mass: MIT Press, 1997.

[19] P. J. Lang, M. M. Bradley, and B. N. Cuthbert, "International affective picture system (IAPS): Technical manual and affective ratings," A6, 1999.

[20] F. G. Ashby, A. M. Isen, and \& U. Turken, "A nueropsychological theory of positive affect and its influence on cognition," Psychol. Rev., vol. 106, no. 3, pp. 529-550, 1999.

[21] G. P. Hodgkinson, J. Langan-Fox, and E. Sadler-Smith, "Intuition: A fundamental bridging construct in the behavioural sciences," Br. J. Psychol., vol. 99, no. 1, pp. 127, Feb. 2008.

[22] D. A. Keim, "Information visualization and visual data mining," IEEE Trans. Vis. Comput. Graph., vol. 8, no. 1, pp. 1-8, Jan. 2002.

[23] S. Attfield, G. Kazai, and M. Lalmas, "Towards a science of user engagement (Position Paper)," p. 8.

[24] T. Bentley and L. Johnston, "EVALUATION USING CUED-RECALL DEBRIEF TO ELICIT INFORMATION ABOUT A USER'S AFFECTIVE EXPERIENCES," p. 10.

[25] P. Vermersch, "Introspection as practice," J. Conscious. Stud., vol. 6, no. 2-3, pp. 17-42, 1999.

[26] A. Light, "Transports of delight? What the experience of receiving (mobile) phone calls can tell us about design," Pers. Ubiquitous Comput., vol. 12, no. 5, pp. 391-400, Jun. 2008.

[27] C. Petitmengin-Peugeot, "The Intuitive Experience," $J$. Conscious. Stud., vol. 6, no. 2-3, pp. 43-77, 1999.

[28] C. Petitmengin, V. Navarro, and M. Le Van Quyen, "Anticipating seizure: Pre-reflective experience at the center of neuro-phenomenology," Conscious. Cogn., vol. 16, no. 3, pp. 746-764, Sep. 2007.

[29] C. Petitmengin and M. Bitbol, "The Validity of First-Person Descriptions as Authenticity and Coherence," J. Conscious. Stud., vol. 16, pp. 363-404, 2009.

[30] "United States gun death data visualization by Periscopic." [Online]. Available: https://guns.periscopic.com. [Accessed: 29-Jun-2018].

[31] D. McCandless, "Plane Truth - Every single commercial plane crash of the last 20 years," Information is Beautiful. [Online].

Available: http://www.informationisbeautiful.net/visualizations/planetruth-every-single-commercial-plane-crash-visualized/. [Accessed: 29-Jun-2018].

[32] V. Braun and V. Clarke, "Using thematic analysis in psychology," Qual. Res. Psychol., vol. 3, no. 2, pp. 77-101, Jan. 2006.

[33] NVIVO. QSR International Pty Ltd., 2018.

[34] A. P. Jha, J. Krompinger, and M. J. Baime, "Mindfulness training modifies subsystems of attention," Cogn. Affect. Behav. Neurosci., vol. 7, no. 2, pp. 109-119, Jun. 2007.

[35] F. A. Olivares, E. Vargas, C. Fuentes, D. Martínez-Pernía, and A. Canales-Johnson, "Neurophenomenology revisited: second-person methods for the study of human consciousness," Front. Psychol., vol. 6, May 2015.
[36] T. Froese, C. Gould, and A. K. Seth, "Validating and Calibrating First- and Second-person Methods in the Science of Consciousness," p. 60. 